\title{
Potentialities of the Numerical Weather Prediction model WRF to produce attenuation statistics in tropical regions
}

\author{
Valentin Le Mire ${ }^{1}$, Xavier Boulanger ${ }^{2}$, Laurent Castanet $^{1}$, Bouchra Benammar ${ }^{2}$, Laurent Féral ${ }^{3}$ \\ ${ }^{1}$ (ONERA): ONERA/DEMR, Federal University of Toulouse, France, Valentin.Le_Mire@onera.fr, Laurent.Castanet@onera.fr \\ 2 (CNES): DSO/RF/ITP, CNES, Toulouse, France, Bouchra.Benammar@cnes.fr, Xavier.Boulanger@cnes.fr \\ ${ }^{3}$ (LAPLACE): GRE, LAPLACE, Federal University of Toulouse, France, lferal@laplace.univ-tlse.fr
}

\begin{abstract}
This paper presents the use of a Numerical Weather Prediction model (the WRF US model from NCAR/NCEP) coupled with an electromagnetic module to create rain attenuation time series and statistical results in a tropical region. Simulated results are compared with experimental data collected within a CNES/ONERA sponsored propagation experiment near Kourou, in French Guiana. Both simulated and experimental Complementary Cumulative Distribution Functions of rain attenuation (CCDF) are presented in an annual and monthly basis. Finally, a brief granulometric study is detailed to better understand the impact of the rain drop size distribution (DSD) on the obtained results.
\end{abstract}

Index Terms-Equatorial and Tropical regions, Ka-Band, WRF, NWP, DSD, propagation, satellite communications.

\section{INTRODUCTION}

In emerging and developing countries in South America, South-East Asia or Africa, satellite communication systems are of great interest compared to ground-based communication systems due to the lack of infrastructure already in place [1]. Because of the saturation of the conventional frequency bands such as $C$ and $K u$ (i.e. under $20 \mathrm{GHz}$ ) for the Fixed Satellite Service, new communication systems for these regions are currently being developed and deployed using higher frequencies, such as $\mathrm{Ka}$ band (20-30 $\mathrm{GHz}$ ) or $Q / V$ band (40-50 GHz). This increase in frequency can also be explained by the wider available bandwidth which is more and more needed with telecommunication applications nowadays. However, above $20 \mathrm{GHz}$, the signal suffers from more severe effects while propagating through the troposphere (attenuation, scintillation, depolarization ...). In tropical and equatorial regions, these effects are even more significant than in temperate regions, because the weather conditions are more adverse. Therefore, specific modelling of the propagation channel is needed in these regions to adapt required fade mitigation techniques or possibly design new ones.

Many studies of the propagation channel have been undertaken during the last two decades at $K u$ and $K a$ frequency bands in temperate regions, but only very few of them have been done for tropical and equatorial regions at frequencies higher than $20 \mathrm{GHz}$ due to the lack of propagation experiment in these regions. To circumvent this lack of experimental data, the use of high resolution Numerical Weather Prediction models (NWP) has been studied in the last few years. ONERA has been a pioneer in this topic and has designed a hybrid meteorologicalelectromagnetic model, coupling the US NWP from NCAR/NCEP named Weather Research and Forecasting (WRF) and an Electromagnetic Module (EMM) to generate attenuation time series [2]. Furthermore, since 2017, CNES and ONERA have been conducting a propagation experiment at $K a$ band at the Guianese Space Centre (GSC), near Kourou (French Guiana), using a beacon receiver for the Amazonas 3 Satellite at $20.2 \mathrm{GHz}$ [3]. This propagation experiment is used to compare the results obtained with the hybrid model WRF-EMM.

Section II details the setup of the Guiana propagation experiment and the global principles of the WRF-EMM model used in this paper. Section III describes the first results obtained with the model in tropical regions. Statistical comparisons with the experimental data collected at GSC are then presented. Finally, Section IV addresses a preliminary granulometric sensitivity analysis from a few existing DSD models introduced in the simulation.

\section{DESCRIPTION OF THE EXPERIMENTAL SETUP}

The propagation experiment mentioned in this paper uses a $K a$ band beacon on board the Amazonas 3 satellite. Launched on February 7, 2013 and located on the geostationary orbit at $61^{\circ} \mathrm{W}$ longitude, the carrier frequency is $20.1995 \mathrm{GHz}$ with a right-hand circular polarization (RHCP).

Since December 2016, CNES has deployed at the GSC a rain gauge, that measures the amount of liquid precipitation, and a beacon receiver pointed towards the Amazonas 3 satellite with respectively elevation and azimuth angles of $78.49^{\circ}$ and $238.36^{\circ}$. These instruments are located at 5.1713 ${ }^{\circ} \mathrm{N}$ latitude and $-52.6862{ }^{\circ} \mathrm{E}$ longitude. The official start of the experiment is January 1, 2017 when both instruments became operational. Two years of measured data are considered in this paper. The availability of the experimental data can be found in Table 1. A more detailed summary of the experiment, as well as the procedure for data processing can be found in [3]. 
TABLE I.

EXPERIMENTAL DATA AVAILABILITY

\begin{tabular}{|c|c|c|}
\hline \multicolumn{3}{|c|}{ Availability (\%) } \\
\hline Instrument & 2017 & 2018 \\
\hline Beacon Receiver & 99.65 & 99.73 \\
\hline Rain Gauge & 99.75 & 100.00 \\
\hline Beacon Receiver + Rain Gauge & 99.58 & 99.46 \\
\hline
\end{tabular}

\section{DESCRIPTION OF THE WRF-EMM MODEL}

The WRF-EMM simulation tool is made of two parts. The first one is a mesoscale NWP model called WRF that can simulate the state of the atmosphere from re-analysis data such as can be found in the ECMWF databases [4]. The second part is an electromagnetic module (EMM) that computes signal attenuation due to rain, clouds, water vapor and oxygen from WRF outputs, using different attenuation models conventionally used today.

\section{A. Introduction to the WRF Model}

From atmospheric data such as temperature, mean sealevel pressure, geopotential, specific humidity, wind profiles ..., WRF solves the Navier-Stokes equations that describe the atmosphere. As long as the input meteorological data are available, WRF simulates the state of the atmosphere over a gridded area. The spatial and time resolutions of the outputs can be configured.

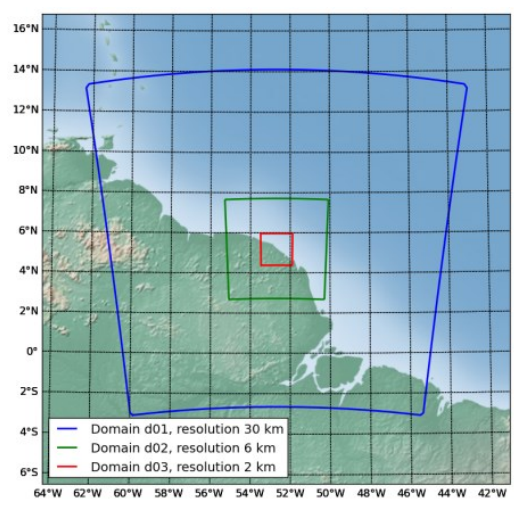

Fig. 1. WRF domains using ERA-Interim data.

In this study, we use the ECMWF ERA-Interim database, available worldwide, with a time resolution of 6 hours and a spatial resolution of $0.75^{\circ} \times 0.75^{\circ}$ (around $84 \mathrm{~km}$ ) to feed the WRF model. The use of ERA-Interim data allows us to configure the WRF model with three grids where the smallest one has a $2 \times 2 \mathrm{~km}$ spatial resolution, as shown on Fig. 1, and with a 5-minute time resolution.

\section{B. Rain Attenuation Computation}

The WRF model produces one output file every 5-minute time-step. These outputs contain a number of meteorological parameters that describe the state of the atmosphere such as temperature, pressure, mixing ratios of the different precipitation types and various thermodynamic variables over the selected grid. The grid is centered over our point of interest, i.e. the location of our propagation experiment and it extends over $150 \times 150 \mathrm{~km}^{2}$ depending on the configuration used [5].

Based on the WRF outputs the EMM module can compute not only rain attenuation time series but also attenuation due to clouds, water vapor and oxygen. However, this paper focuses on rain attenuation, i.e. the major contributor to total tropospheric attenuation at these frequencies.

The procedure to compute rain attenuation over a given satellite radio-link is given in [2] and [6]. It is summarized in Fig. 2. After calculating the specific attenuation due to rain $\gamma_{r}(\mathrm{~dB} / \mathrm{km})$ for each pixel of the 3D grid at a given frequency and for given elevation and azimuth angles, an integration of $\gamma_{r}$ on the radio path gives the total attenuation suffered by the signal.

The specific attenuation due to rain can be calculated as follows:

$$
\gamma_{r}=4.3 \cdot 10^{-3} \int_{0}^{D_{\max }} \sigma_{\text {ext }}(D) N(D) d D
$$

where $\sigma_{\text {ext }}(D)$ is the total extinction cross-section of a raindrop of diameter $\mathrm{D}$ (in $\mathrm{mm}$ ), $N(D)$ is the drop-size distribution (DSD) and $D_{\max }$ is the diameter of the largest raindrop considered.

While $\sigma_{\text {ext }}(D)$ is given by the Mie theory, $N(D)$ must be calculated with a Rain DSD model such as Marshall-Palmer (MP) or Ulbrich-Atlas (UA) [7] - [8]. In this paper, the MP DSD model is the one used if it is not mentioned otherwise.

\section{ANNUAL AND MONTHLY Statistical ANALYSIS}

Once the synthetic rain attenuation time series has been computed, a statistical analysis is undertaken using Complementary Cumulative Distribution Functions (CCDF) to see the behavior of the rain attenuation over 2 full years. Fig. 3 shows the comparison between the experimental data and the simulated results using WRF-EMM. The rain attenuation CCDF obtained with the prediction method given by Rec. ITU-R P.618-13 over 2017, 2018 [9] is also represented.

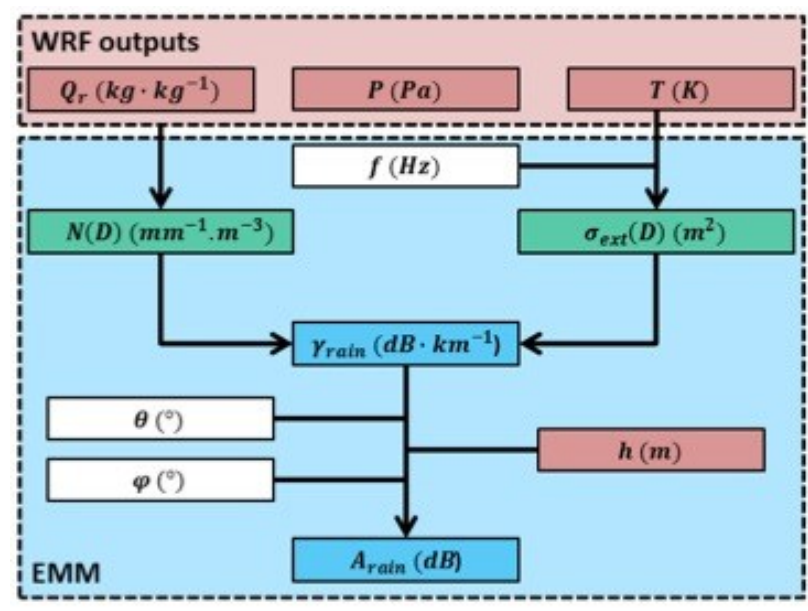

Fig. 2. Overview of rain attenuation computation using WRF outputs [6]. 


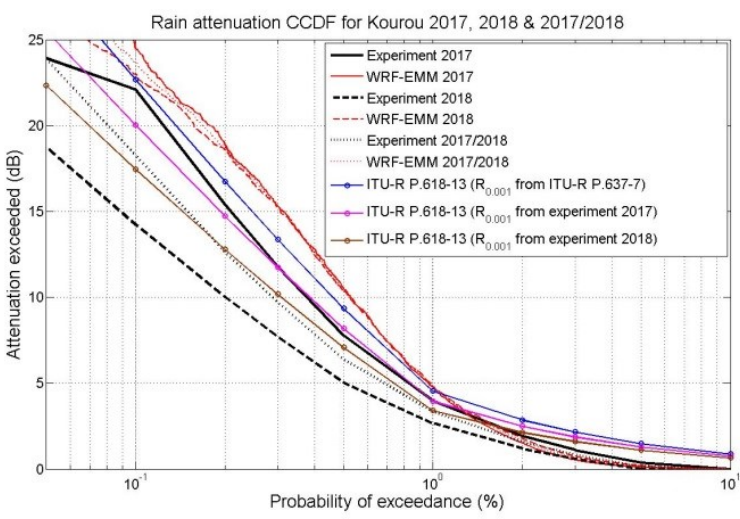

Fig. 3. Annual and bi-annual CCDF of rain attenuation at $20.2 \mathrm{GHz}$.

We can first notice that the rain attenuation CCDFs obtained with the WRF-EMM model compare reasonably well with the experimental statistics, even though the model overestimates the rain attenuation for low percentages of time and underestimates it for high percentages of time.

A brief comparison with the rain attenuation obtained with the prediction method in Rec. ITU-R P.618-13 is undertaken. The prediction method uses the rainfall rate values exceeded for $0.01 \%$ of the year $\left(\mathrm{R}_{0.01}\right)$ as an input. In Fig.3, we have used three different $\mathrm{R}_{0.01}$ : the first one calculated with the ITU-Rec P.837-7 while the second and the third are experimental values for years 2017 and 2018 [10]. We observe an overestimation of the rain attenuation for high percentages of time but a better behavior than WRFEMM for low percentages of time.

We can conclude with this preliminary comparison that the WRF-EMM model gives quite reasonable results that should probably be improved by tuning the model configuration (especially the DSD). To complete this statistical analysis, the monthly CCDFs for the year 2017 have been computed to see if the synthetic statistics produced by WRF-EMM simulations match the experimental CCDFs. The results are shown in Fig.4 and Fig.5.

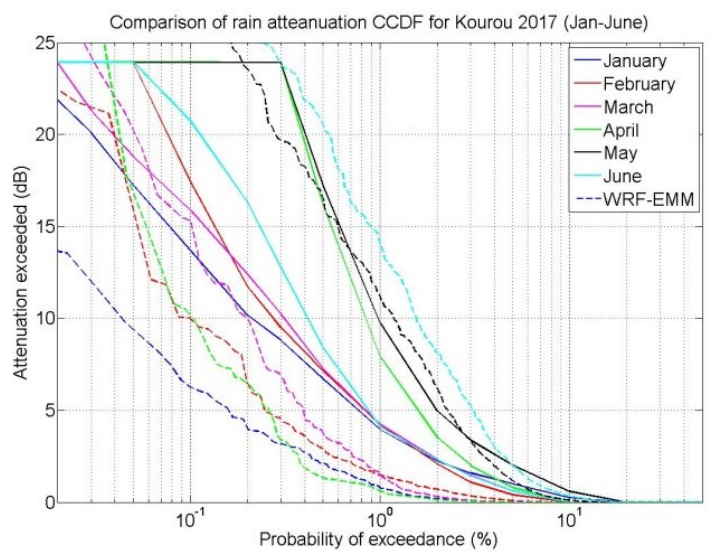

Fig. 4. Monthly CCDF of rain attenuation for January to June 2017, from WRF-EMM simulations (dotted lines) and from the experimental data (full lines).

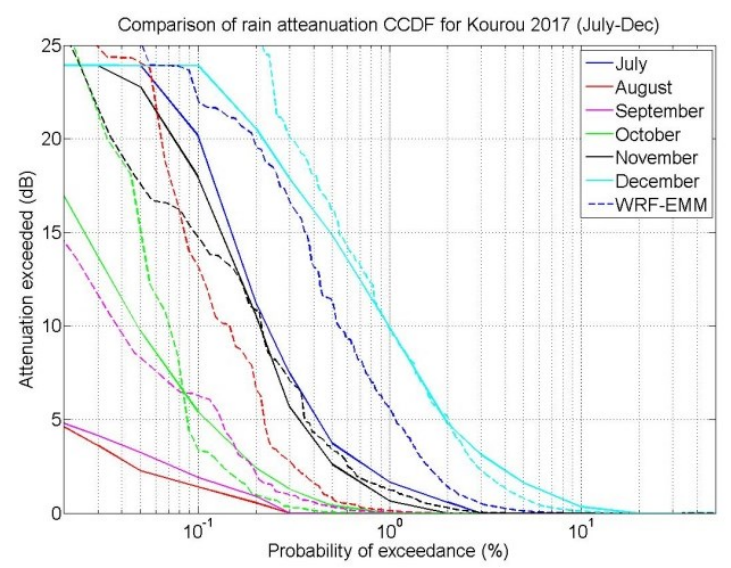

Fig. 5. Monthly CCDF of rain attenuation for July to December 2017, from WRF-EMM simulations (dotted lines) and from the experimental data (full lines).

The synthetic statistics obtained from WRF-EMM for the months of May, November and December are very close to the experimental CCDFs. However, for the rest of the year the results do not coincide. This inadequacy for certain months should be linked to the rainy and dry seasons that occur in Kourou. Indeed, in this tropical climate, two rainy seasons (from April to June and from December to January) as well as one dry season (from August to October) can be identified. However, as seen in Fig.5, the rainy and dry seasons do not match any kind of behavior seen on the monthly CCDFs. Here the short duration of the experiment needs to be taken into account. Indeed, for one month the sample used may be too small to accurately compare statistics such as CCDF.

Furthermore, we can notice two interesting though different behaviors of the WRF-EMM model. During the most important rainy season that lasts from April to June, we can see on Fig. 4 that WRF-EMM seriously overestimates the rain attenuation for the month of June. On the contrary, we observe a strong underestimation of WRF-EMM for the month of April. However, when we look at the rain accumulation computed by WRF-EMM it overestimates the data collected by the rain gauge for both months. This would led us to believe that the rain accumulation is not the most important criteria when it comes to rain attenuation in tropical regions. A granulometric sensitivity analysis is overseen in section $\mathrm{V}$ to see if the difference obtained in the results could come from an inadequacy between the experimental DSD and the MP model.

\section{GRANULOMETRIC ANALYSIS}

This section deals with the preliminary granulometric sensitivity analysis that was undertaken for the month of April 2017 in Kourou. For this analysis, only two models of DSD have been used, the Marshall-Palmer and UlbrichAtlas models. Both models represent the DSD as a truncated gamma function:

$$
N(D)=N_{0} D^{\mu} e^{-\Lambda D}
$$


where $\mathrm{D}$ is the diameter of the raindrops in $\mathrm{mm}, N_{0}$ a constant in $m^{-3} \mathrm{~mm}^{-1-\mu}, \mu$ the dimensionless shape parameter and $\Lambda$ the slope parameter in $\mathrm{mm}^{-1}$ that can be calculated using the WRF output describing the rain water mixing ratio [7] $-[8]$.

The case $N_{0}=8000, \mu=0$ corresponds to the MP distribution while UA describes a dependency between the two constants:

$$
N_{0}=6730.10^{-\mu} e^{3.75 \mu}
$$

For this analysis, we compare the rain attenuation CCDF obtained with the MP model and the UA model for different values of $\mu$ from -2 to 2 . The results are shown in Fig. 6 .

The results show certain sensitivity to the DSD model used to generate the attenuation time series. However, between the different models, we can only see a difference of a few decibels on the rain attenuation CCDF.

Though, the use of different DSD models can modify by a few decibels the CCDF calculated, we can only conclude that it does not have a sufficient impact to explain the discrepancy between the WRF-EMM model and the experimental data seen on Fig. 4 and Fig. 5.

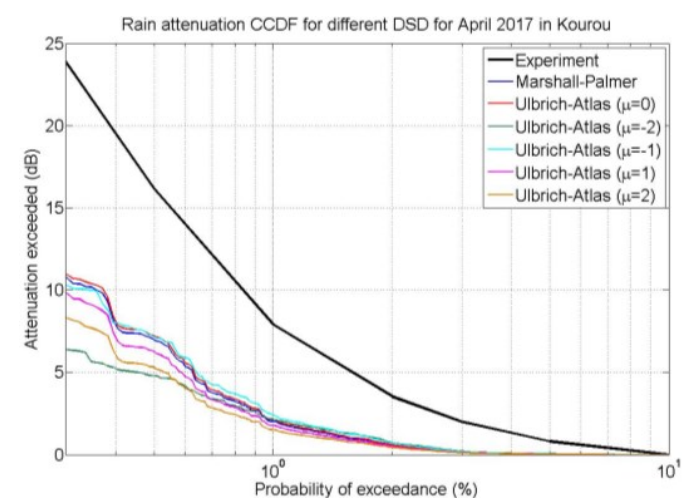

Fig. 6. Comparison of the rain attenuation CCDF for April 2017 in Kourou using different DSD models.

\section{CONCLUSION}

This paper presents the use of a NWP model called WRF combined with an electromagnetic module to generate rain attenuation time series in tropical and equatorial regions. Using the propagation experiment conducted by CNES and ONERA at the GSC in Kourou, the synthetic results are compared to the two full years of experimental data collected with a rain gauge and a beacon receiver at 20.2 $\mathrm{GHz}$.

The statistical analysis through annual and bi-annual rain attenuation CCDF shows reasonable results, with an overestimation of the rain attenuation for low percentages of time and an underestimation for high percentages of time. The comparison is then undertaken on a monthly basis to try and identify a seasonal correlation. Only for the months of May, November and December, does the synthetic CCDF show good adequacy with the experimental data.
Furthermore, no seasonal correlation could be found, as the two rainy seasons extend from April to June and from December to January.

Finally, to try and explain the difference between the experimental and synthetic results, a granulometric sensitivity analysis is showed. Though, a difference of a few decibels between each DSD model used can be observed on the $\mathrm{CCDF}$, the impact is not significant enough to explain the discrepancy between the synthetic and experimental monthly CCDF.

These results lead us to conclude that the meteorological physical models inside the NWP WRF could be the cause of the discrepancy and should be investigated more thoroughly.

\section{REFERENCES}

[1] L. Castanet et al., "Assessment of radiowave propagation for satellite communication and navigation systems in tropical and sub-tropical areas" Final report for the European Space Agency under ESTEC Contract No.18278/04/NL/US, Juin 2017.

[2] M. Outeiral García, N. Jeannin, L. Féral and L. Castanet, "Use of WRF model to characterize propagation effects in the troposphere," 2013 7th European Conference on Antennas and Propagation (EuCAP), Gothenburg, 2013, pp. 1377-1381.

[3] X. Boulanger, B. Benammar and L. Castanet, "Propagation Experiment at Ka-Band in French Guiana: First Year of Measurements," in IEEE Antennas and Wireless Propagation Letters, vol. 18 , no. 2, pp. 241-244, Feb. 2019

[4] W. C. Skamarock et al., "A Description of the Advanced Research WRF Model Version 3" NCAR Technical Notes NCAR/TN-475+ STR, June 2008

[5] G. Fayon, L. Féral, L. Castanet, N. Jeannin, X. Boulanger, "Use of WRF to Generate Site Diversity Statistics in South of France", persented at 32nd URSI GASS, Montreal, 19-26 August 2017

[6] G. Fayon, "Modélisation Statistique de la Diversité Multi-Site aux Fréquences Comprises entre 20 et $50 \mathrm{GHz}$ " $\mathrm{PhD}$ thesis, University of Toulouse 3 Paul Sabatier, 2017.

[7] J. Marshall, W. McK. Palmer, "The Distribution of Raidrops with Size" Journal of Meteorology, vol. 5, no. 4, pp. 165-166, August 1948

[8] C. W. Ulbrich; D. Atlas, "Rainfall Microphysics and Radar Properties: Analysis Methods for Drop Size Spectra" Journal of Applied Meteorology, vol. 37, pp. 912-923, September 1998

[9] "Propagation data and prediction methods required for the design of Earthspace telecommunication systems" ITU-R, Geneva, Switzerland, Recommendations P.618-13, December 2017.

[10] "Characteristics of precipitation for propagation modelling" ITU-R, Geneva, Switzerland, Recommendations P.837-7, June 2017. 